\title{
Ciclo de vida y aspectos poblacionales de Edessa aff. aulacosterna Stal, 1872 (Heteroptera: Pentatomidae) chinche del fruto del camu camu (Myrtaceae) en zona de restinga, Ucayali, Perú
}

\author{
José IANNACONE ${ }^{1}$, Diana PEREZ ${ }^{2,3}$ y Alfredo TUEROS ${ }^{1,4}$
}

\begin{abstract}
RESUMEN
El camu camu es uno de los frutales nativos con gran potencial económico para la agroindustria y agro exportación. Edessa es una de las plagas del camu camu que provoca sequedad en los brotes, y en los frutos una mancha decolorada con círculos concéntricos bien marcados y un punto central al alimentarse. El objetivo del presente trabajo fue determinar el ciclo biológico bajo condiciones de laboratorio y la fluctuación poblacional de Edessa aff. aulacosterna "Chinche del fruto del camu camu" durante enero a noviembre del 2004 en el cultivo de camu camu, en parcelas en desarrollo y producción ubicadas en áreas de restinga inundables en Pucallpa, Ucayali, Perú. Se colectaron huevos, ninfas y adultos procedentes del distrito de Yarinacocha, Pucallpa, Ucayali, Perú para iniciar la crianza artificial. El tiempo de duración del huevo al I estadio ninfal fue 5,1 días. Se encontró un 94,6\% de eclosión de los huevos, variando entre 12 hasta 14 huevos por postura. El tiempo transcurrido desde el huevo hasta II estadío fue de 37,3 días y del III al V estadío ninfal fue de 81,9 días. El porcentaje de mortalidad del primer estadio ninfal a adulto fue de 98,1\%. Los adultos colectados se localizaron preferentemente sobre las ramas y el tallo de la planta, mientras que las ninfas se encontraron en los brotes tiernos. Con relación a su fluctuación poblacional, solo se registró posturas a lo largo del año en parcelas en producción. No se encontraron diferencias entre ninfas I a V, y adultos de Edessa aff. aulacosterna, entre plantaciones en producción y desarrollo de camu camu. Solo se encontró diferencias entre la época seca y lluviosa en las ninfas de III a V estadio en las plantas de camu camu en desarrollo. Edessa aff. aulacosterna presentó mayormente una distribución contagiosa. El adecuado conocimiento de la bioecología nos permite mejorar la estrategia de control de esta plaga.
\end{abstract}

PalabraS-ClaVE: Amazonia, Camu-camu, Ciclo biológico, Edessa, Pentatomidae.

\section{Life cycle and population aspects of camu camu fruit stink bug Edessa aff. aulacosterna Stal, 1872 (Heteroptera: Pentatomidae) in restinga zone, Ucayali, Peru}

\begin{abstract}
Camu camu is one of the native fruits with a great economic potential for agroindustry and agro exportation. Edessa is one camu camu pest that produces in dry buds and fruits with a mark decolorated with concentric circles well marked and a central point when they feed. The aim of the current research was to determinate the biological cycle under laboratory conditions and population fluctuations of Edessa aff. aulacosterna. "Camu camu fruit stink bug" during January to November, 2004 in camu-camu culture, in development and production parcels located in flooded areas of restinga in Pucallpa, Ucayali, Peru. Eggs, nymphs and adults were colected to begin artificial rearing proceeding of distrit of Yarinacocha, Pucallpa, Ucayali, Peru. Development time of eggs to $1^{\text {th }}$ nymphal instar was 5.1 days. $94.6 \%$ of egg hatchability, varied between 12 until 14 egg-laying was found. The time passed since eggs until III nymphal instar was 37.3 días and of III to V nymphal instar was de 81.9 days. Percentage of mortality of $2^{\text {nd }}$ nymphal instar to adult was $98,1 \%$. Adults collected were localized mainly on branches and stem of the plants, while nymphs were found in delicated buds. In relation to seasonal populations only were registered eggs laying throught the year in parcels in production. Differences between nymphs I to $\mathrm{V}$ and adults of Edessa aff. aulacosterna, in production and development plantations were not found. Only differences in dry and wet period in III to V nymphs instar in development camu camu plants were found. Edessa aff. aulacosterna mainly presented a contagious distribution. Suitable knowledge of bioecology of Edessa aff. aulacosterna permit improves strategy of pest control.
\end{abstract}

KEY WORDS: biological cycle, Amazonia, camu camu, Edessa, Pentatomidae.

1 Laboratorio de Ecofisiología Animal. Facultad de Ciencias Naturales y Matemáticas. Universidad Nacional Federico Villarreal. Calle San Marcos 383, Pueblo Libre, Lima, Perú. E-mail: joseiannacone@gmail.com. ${ }^{2}$ Instituto de Investigaciones de la Amazonía Peruana (IIAP) - Ucayali. Jr. Progreso 102, Pucallpa, Ucayali, Perú. ${ }^{3}$ E-mail: dperez@iiap.org.pe ${ }^{4}$ E-mail: alfredo_tueros@hotmail.com 


\section{INTRODUCCIÓN}

El camu camu Myrciaria dubia H.B.K. Mc Vaugh (Myrtaceae) es uno de los frutales nativos amazónicos con gran potencial económico para la agroindustria y agroexportación por su alto contenido de ácido ascórbico, encontrándose entre 410 a $3.253 \mathrm{mg}$ por $100 \mathrm{~g}$ de pulpa (Villachica, 1996; Rodrigues et al., 2001; Nascimento et al., 2003).

La región Ucayali, localizada en la zona sur de la Amazonía Peruana, por su especial ubicación geográfica y fisiografía posee grandes condiciones para el desarrollo óptimo de este cultivo; como consecuencia del incremento de las áreas de cultivo de camu camu, como ocurre en cualquier otro cultivo monoespecífico se viene observando la aparición de numerosas plagas (Pinedo et al., 2001). En la actualidad se tienen reportados 70 especies de insectos plagas para el camu camu (Delgado y Couturier, 2004). En el listado de plagas registradas en la región Ucayali, Edessa sp. (= Edessa aff. aulacosterna Stal, 1872) (Hemiptera: Pentatomidae), conocido como "chinche del fruto del camu camu", se encuentra en mayor densidad en las restingas inundables, que tienen a su alrededor bosque primario o en la maleza adyacente a las parcelas (Pinedo et al., 2001). Edessa Fabricius, 1803 es de amplia distribución en el Neotrópico (Panizzi, 2002; Barcellos y Grazia, 2003; Silva et al., 2004).

Pinedo et al. (2001) mencionan que el adulto de Edessa sp. es de color verde, la parte membranosa del ala presenta manchas de color marrón y mide 12 a $14 \mathrm{~mm}$ de largo. La hembra oviposita sobre las hojas. Los huevos miden entre 2,0 a 2,5 mm de diámetro, son de color verde y se oscurecen poco a poco hasta la salida de las ninfas, y permanecen agrupadas hasta el segundo estadío. Edessa sp. provoca que los brotes se sequen, y en los frutos una mancha decolorada con círculos concéntricos bien marcados y un punto central al alimentarse. La acción mecánica producida por la picadura del chinche induce a que el fruto se licue, perdiendo el contenido y desnaturalizando el ácido ascórbico.

Teniendo en cuenta la escasa información sobre esta plaga con respecto a su ciclo biológico y comportamiento, el presente trabajo tuvo como objetivo conocer su ciclo biológico bajo condiciones de laboratorio y determinar su fluctuación poblacional de Edessa sp. en parcelas de camu camu en crecimiento y desarrollo ubicadas en áreas de restinga inundables en Pucallpa, Ucayali, Perú. La especie que fue registrada como Edessa sp fue identificada por el Dr. José Antônio Marin Fernandes, UFPA, Brasil y se trata de una especie nueva próxima a Edessa aulacosterna Stål, 1872.

\section{MATERIALES Y MÉTODOS}

\section{CICLO BIOLÓGICO DE EDESSA AFF. AULACOSTERNA}

La crianza se desarrolló en el Laboratorio de Entomología del Instituto de Investigaciones de la Amazonía Peruana (IIAP), Ucayali, Perú entre Enero a Noviembre del 2004. Las condiciones del Laboratorio presentaron una temperatura de $25,4 \pm 1,8^{\circ} \mathrm{C}$ y una humedad relativa de $77,4 \pm 2,7 \%$.

\section{COLECTA}

Se colectaron procedente del campo una postura con 14 huevos, 29 ninfas entre el I y II estadío, 62 ninfas entre el III y V estadío y 30 adultos, los cuales sirvieron de base para la crianza artificial. Los adultos colectados se localizaron preferentemente sobre las ramas y el tallo de la planta, mientras que los diferentes estadios ninfales se encontraron en los brotes tiernos. Las colectas se realizaron entre Enero y Febrero del 2004 en las parcelas de la Empresa Agrícola San Juan, ubicadas en los km 11 y 13 de la Carretera Federico Basadre (C.F.B.), en las parcelas del caserío San Juan y Padre Bernardo del distrito de Yarinacocha, en las parcelas de la Ing. Rita Riva ubicada en el km 10 y $4 \mathrm{Km}$ al interior, y en el vivero del IIAP C.F.B. km 12,400, Pucallpa, Ucayali, Perú. La captura manual de las ninfas y los adultos se realizó entre las 8:00 h hasta las 14:00 h (Malaguido y Panizzi, 1999). Una vez colectados los insectos se procedió a colocarlos en recipientes de plástico de $30 \mathrm{~cm} \times 15 \mathrm{~cm} \times 8 \mathrm{~cm}$, acondicionados con una base de papel toalla humedecida, con frutos maduros, brotes tiernos y una cubierta de tela de holganza a fin de protegerlos de la desecación durante el transporte al Laboratorio (Noda y Kamano, 2002). Los ejemplares colectados se trasladaron al laboratorio de Entomología del IIAP-Ucayali con la finalidad de iniciar la crianza artificial.

\section{CRIANZA}

Los adultos colectados en campo se separaron por sexo y se dispusieron en parejas por jaula para la obtención de las posturas (Sosa-Gomez y Moscardi, 1998; Perioto et al., 2004). Cada jaula consistió en dos envases plásticos de 11,5 $\mathrm{cm}$ de diámetro y $12,8 \mathrm{~cm}$ de longitud, colocados uno encima de otro. En la parte superior se protegió con una tela de tul para permitir el pasaje del aire. En el envase inferior se colocó agua y se cerró con una tapa plástica, en la parte central de la tapa se aperturó un orificio, que sirvió para disponer los brotes tiernos de camu camu, los que fueron utilizados como alimento y medio de oviposición. Con una pipeta se aplicó ad libitum gotas de miel de abeja sobre los brotes y se complementó con un trozo de algodón embebido en agua (Perioto et al., 2004). Diariamente, los huevos que fueron 
depositados por las hembras sobre los brotes fueron removidos y trasladados a nuevas jaulas de iguales características a las citadas anteriormente (Riba et al., 2003). Una vez que los huevos estuvieron próximos a eclosionar, se les reconoció por la aparición de puntos de color rojo. Para cada estado de desarrollo: huevo, ninfas I al V, y adulto se evaluó la duración de cada una de las etapas (en días), su tamaño promedio (en $\mathrm{mm}$ ) y algunas características morfológicas. Durante toda la cría de Edessa, se les suministró brotes tiernos, miel de abeja y algodón humedecido, los cuales fueron cambiados tres veces por semana.

\section{FLUCTUACIÓN POBLACIONAL DE Edessa aff. aulacosterna EN PARCELAS DE CAMU-CAMU}

El monitoreo de fue realizado en las parcelas de camu camu de los caseríos San Juan (3,5 ha en producción y 3,5 ha en crecimiento), ubicado a $30 \mathrm{~min}$ por río del puerto de Yarinacocha y en el caserío Padre Bernardo (1,5 ha en producción y 1,5 ha en desarrollo), ubicado a $1 \mathrm{~h}$ y $15 \mathrm{~min}$ por río del puerto de Yarinacocha, a la margen izquierda del lago de Yarinacocha, Ucayali, Perú durante Febrero a Noviembre del 2004.

Con la finalidad de determinar la presencia de la plaga en cada zona, se evaluaron mensualmente 20 plantas.ha al azar, recorriendo el campo en forma de "X". Se dividió cada planta en tercios: superior (TS), medio (TM) e inferior (TI). Se revisó minuciosamente la presencia de Edessa aff. aulacosterna y se contaron individuos en sus diferentes estados de desarrollo (huevos, ninfas I al V, y adultos) (Todd, 1989; Panizzi y Vivan, 1997; Correa-Ferreira y de Azevedo, 2002; Jones y Westcot, 2002). Se empleó la prueba de t de Student para datos pareados para determinar si existían diferencias entre el número de individuos colectados mensualmente en los estadios ninfales I a II, III a V, y adultos en parcelas en

Tabla 1 - Duración en días de los diferentes estados de desarrollo de Edessa aff. aulacosterna bajo condiciones de laboratorio en Pucallpa, Ucayali, Perú.

\begin{tabular}{lcllll}
\hline $\begin{array}{l}\text { Estados de } \\
\text { desarrollo }\end{array}$ & $n$ & $\mathrm{X} \pm \mathrm{DE}$ (días) & $\begin{array}{c}\text { Rango en } \\
\text { días }\end{array}$ & $\begin{array}{c}\text { Mortalidad } \\
(\%)\end{array}$ & $\begin{array}{l}\text { Mortalidad } \\
\text { acumulada }\end{array}$ \\
\hline Huevo & 113 & $5,3 \pm 0,4$ & $5-6$ & $5,3^{*}$ & $5,3^{*}$ \\
\hline Ninfa I & 107 & $5,1 \pm 0,3$ & $5-6$ & 63,5 & 65,4 \\
\hline Ninfa II & 39 & $26,9 \pm 4,6$ & $19-35$ & 46,1 & 81,4 \\
\hline Ninfa III & 21 & $34,4 \pm 12,4$ & $18-82$ & 47,6 & 90,2 \\
Ninfa IV & 11 & $24,9 \pm 5,1$ & $17-33$ & 54,5 & 95,5 \\
\hline Ninfa V & 5 & $22,6 \pm 2,3$ & $19-25$ & 60 & 98,2 \\
Adulto & 2 & $44,5 \pm 1,3$ & $43-46$ & - & - \\
Total & & 119,2 & $83-187$ & & \\
\hline
\end{tabular}

$\mathrm{n}=$ número de ejemplares al inicio de cada fase. $\mathrm{X}=$ promedio. $\mathrm{DE}=$ Desviación estándar. * $=$ Porcentaje no eclosionados. producción y desarrollo. En adición, se usó la prueba de $\mathrm{t}$ de Student para determinar si existían diferencias entre la época seca (mayo a octubre del 2004) y lluviosa (febrero a abril, y noviembre del 2004) para los estadios ninfales I a II, III a V, y adultos en parcelas en producción y desarrollo de camu camu. Para todos los casos se empleó la prueba de Levene para verificar la homogeneidad de las varianzas. Los resultados obtenidos de la fluctuación poblacional de Edessa aff. aulacosterna fueron evaluados utilizando la distribución de Poisson mediante el estadístico Chi-cuadrado y el índice de varianza/media (ID) para determinar si su distribución es al azar, uniforme o agregada (Zar, 1996).

\section{RESULTADOS Y DISCUSIÓN}

\section{CICLO BIOLÓGICO}

\section{HUEVO}

De 9 posturas evaluadas obtenidas bajo condiciones de crianza, el 100\% fueron depositados sobre el haz de las hojas tiernas. De 113 huevos evaluados el 94,6\% eclosionaron. Se ha observado para el pentatomido Nezara viridula (Linnaeus, 1758) que el porcentaje de eclosión de los huevos fue menor (entre $53,2 \%$ a $80,4 \%$ ). El número de posturas varió de 12 hasta 14 huevos dispuestos en dos hileras paralelas, semejante a lo observado en otras especies de Edessa (Sánchez et al., 1999). El 77,7\% de las posturas contuvo 14 huevos, con una media de 13,6 \pm 0,9. Serra y La Portana (2001) han encontrado en Piezodorus guildinii (Westood, 1873), un promedio de 9,5 huevos por postura. El tamaño promedio de los huevos fue de $1,4 \mathrm{~mm}$ de diámetro y el tiempo de duración de los huevos osciló entre 5 y 6 días con un promedio de 5,3 \pm 0,4 días (Tabla 1). Estos resultados difirieron a lo señalado por Delgado (datos no publicados), quien señala 7 días para $E$ dessa sp., y superior a lo registrado por Sánchez et al. (1999) para Edessa meditabunda (Fabricius, 1794) con 4,7 dias. Al inicio los huevos son de color verde cristalino, presentando pequeñas rugosidades en la superficie y a medida que se acerca la eclosión estos se tornan de color verde oscuro (Figura 1a,b). El tiempo de eclosión duró generalmente de 1 a $2 \mathrm{~h}$.

\section{NINFA I}

De una población de ninfas del I estadío ( $\mathrm{x}=107)$ el tamaño promedio fue de $1,9 \mathrm{~mm}$. Delgado (datos no publicados) menciona un promedio de 1,95 para la misma especie, y con una duración promedio de 5,1 \pm 0,3 días (Tabla 1) con un rango que difiere con respecto a la de E. meditabunda que registró 3 y 11 días (Sánchez et al., 1999). El 36,5 \% de ninfas I pasaron al II estadío (Tabla 1). Las ninfas emergidas permanecieron inmóviles ya que tienen una fuerte tendencia a permanecer agregadas alrededor o sobre los restos de los huevos durante el tiempo que dura el I estadío ninfal, no se alimentaron aunque tuvieron cerca la fuente de alimento, 
a.

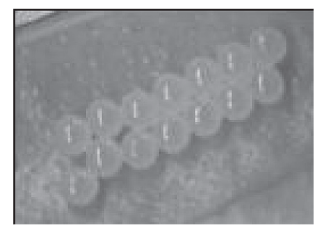

c.

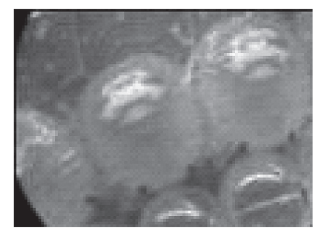

e.

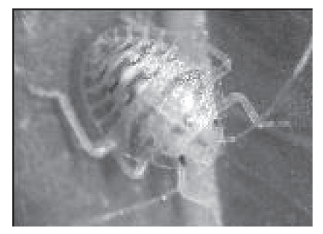

g.

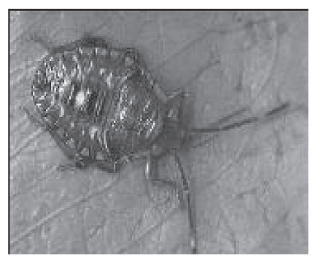

i.

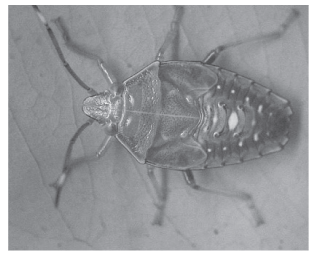

b.

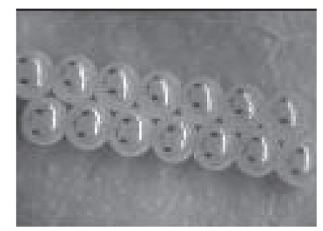

d.

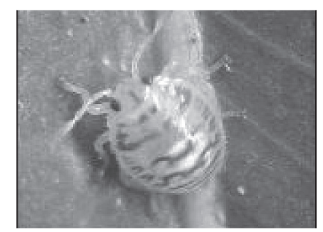

f.

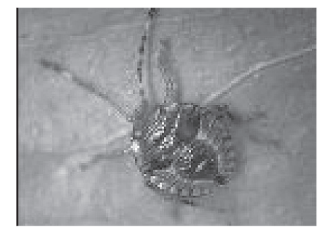

h.

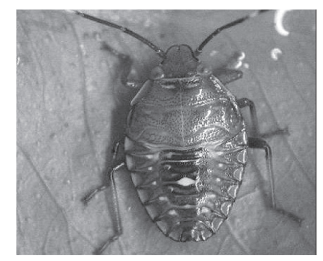

j.

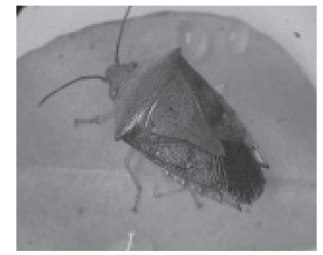

Figura 1- Edessa aff. aulacosterna "chinche del fruto del camu camu". a,b. Posturas recién ovipositadas y previa a la eclosión. c,d. Ninfas I estadio recién emergidas y cerca de su primera muda. e,f. Ninfa al inicio del II estadío y con cutícula quitinizada. g. Ninfa del III estadío. h. Ninfa del IV. i. Ninfa del V. j. Adulto.

similar comportamiento fue observado en E. meditabunda (Sánchez et al., 1999). En el caso de $N$. viridula no se alimentaron (Todd, 1989). Sánchez et al. (1999), mencionan que las ninfas de $P$. guildinii permanecieron cerca de los restos de los huevos y no se alimentaron, mientras que en E. meditabunda se alimentaron muy poco. Al nacer el cuerpo y las extremidades de las ninfas I presentaron una coloración verde claro con una mancha circular con franjas de color crema y de color rojo sobre la parte dorsal y los ojos de color rojo. En el transcurso de los días el cuerpo se torna de color blanco con las manchas de color roja más pronunciadas y en mayor número sobre el cuerpo, presentando forma semicircular (Figura $1 \mathrm{c}, \mathrm{d}$ ).

\section{NINFA II}

Las ninfas del II estadío $(\mathrm{x}=39)$ presentaron un tamaño promedio de 3,10 $\mathrm{mm}$ de largo y un tiempo de vida en este estadio que osciló entre 19 y 35 días con un promedio de 26,9 \pm 4,6 días, lo cual difiere con E. meditabunda que registra 8,9 días (Sánchez et al., 1999). Dos ninfas permanecieron en ninfa II hasta los 42 y 48 días, respectivamente. Asimismo, Delgado (datos no publicados), registró en Edessa sp. en camu camu una longitud promedio de $3,5 \mathrm{~mm}$ de largo y una duración de 17 días. Al iniciarse el II estadío, el cuerpo del insecto presentó una coloración blanco hialino con manchas de color rojo, luego la cutícula se quitinizó y a medida que esto sucede el cuerpo adquiere una tonalidad ámbar-negro y pequeñas tonalidades rojo y blanco (Fig. 1e,f). Los tres pares de patas de color ámbar, con las primeras articulaciones de los dos pares de patas posteriores ligeramente oscuras; las antenas de color ámbar oscuro con el último segmento de color blanco y negro. Las ninfas del II estadío se caracterizaron por presentar mayor actividad que los demás estadíos, constantemente estuvieron en movimiento, desplazándose por toda la planta. $\mathrm{Al}$ agregarse forman grupos de 4 y 5 ninfas presentando forma semicircular. Del total de la población de ninfas solo $21(53,9 \%)$ pasaron al III estadío. Durante el estadío II las ninfas se dispersan desde los restos de los huevos hacia la fuente de alimento, generalmente en esta fase se presenta una alta mortalidad para muchas especies de pentatómidos; el comportamiento gregario disminuye a medida que avanza el desarrollo.

\section{NINFA III}

Las 21 ninfas del III estadío presentaron un tamaño promedio de 4,5 mm de largo, lo cual coincide con lo señalado por Delgado (datos no publicados) con 4,5 mm. Las ninfas de III estadío tuvieron un promedio de 34,4 $\pm 12,4$ días antes de pasar al estadio IV (Tabla 1g), lo que difiere por lo observado por Delgado (datos no publicados) quien registró 8-9 días para la misma especie. En este estadío los colores son muy similares a la del II estadío pero más definidos, las manchas de color blancas se tornan de color crema presentando simetría en la parte dorsal, la región abdominal adquiere una coloración cremosa y ligeramente verde cerca de los bordes, sobre el dorso aparecen manchas semicirculares, en el centro de estas una mancha más grande de forma alargada todas de color crema y sobre la cabeza dos franjas pequeñas y una franja grande de color blanco cremoso que une los ojos; en este estadío presenta el cuerpo más alargado que el estadío anterior y de forma elipsoidal, adquiriendo la forma característica de los pentatómidos (Figura 1g). No se observó vestigios alares. Asimismo, Delgado (datos no publicados) refiere que el cuerpo presenta una tonalidad semejante a la del III estadío. Las ninfas del estadío III de Edessa pueden agregarse en pequeños grupos, cerca de la fuente de alimento como ocurre con $N$. viridula. 


\section{NINFA IV}

Se evaluaron 11 ninfas del IV estadío, cuyo tamaño promedio fue de $7,9 \mathrm{~mm}$ (Figura 1h). Se alimentaron con miel de abeja, y el tiempo de duración osciló entre 17 a 33 días con un promedio de $24,9 \pm 5,1$ días, lo cual difiere con lo observado por Delgado (datos no publicados) quien registró 17 días para la misma especie y con Sánchez et al. (1999), con 7,2 días para E. meditabunda. En el estadío IV se presenta una fuerte competencia por el alimento, por lo que las ninfas se dispersan. Solo 5 ninfas pasaron al V estadío.

\section{NINFA V}

Las cinco ninfas del $V$ estadío se alimentaron con miel de abeja, demoraron entre 19 a 25 días para pasar al estado adulto (Tabla 1). Sánchez et al. (1999) registró 10,96 días para E. meditabunda. La ninfa midió $10,9 \mathrm{~mm}$ de largo, similar a lo registrado por Delgado (datos no publicados) para la misma especie. En este último estadío ninfal, se observó la formación de las alas. Se observó la formación de vestigios alares en este estadío; presentando una coloración más clara con respecto a los anteriores estadíos y permaneciendo con tonos de color rojo y verde claro por espacio de 12 días, para luego cambiar a una tonalidad verde en todo el cuerpo, por espacio de 6 días hasta llegar al estado adulto (Figura 1i). El estadío $\mathrm{V}$ puede ser considerado el más crítico dentro del desarrollo ninfal, en este periodo el insecto necesita un alimento de alta calidad nutricional para que el futuro adulto exprese el máximo potencial reproductivo. La mortalidad en esta fase es similar a la que ocurre en el estadío II (Sánchez et al., 1999). Serra y La Portana (2001) han encontrado en P. guildinii, una mortalidad ninfal de $60,1 \%$, siendo mayor durante el segundo estadio.

\section{ADULTOS}

Los adultos $(\mathrm{x}=2)$ fueron alimentados con miel y algodón húmedo. La longevidad fue de 43 días (hembra) y 46 días (macho); Sánchez et al. (1999) indican para E. meditabunda una longevidad menor de 33 días para los machos y 34,7 dias para las hembras (Figura 1j). Serra y La Portana (2001) han encontrado en $P$. guildinii, una longevidad para los adultos de 60 y 78,5 días para machos y hembras, respectivamente. En adición, para adultos de $P$. guildinii se ha encontrado una longevidad entre 22,5 a 25,3 días (Panizzi et al., 2000). En $N$. viridula se ha observado una menor longevidad de los chinches adultos (Noda y Kamano, 2002).

\section{FLUCTUACIÓN POBLACIONAL DE Edessa aff. aulacosterna \\ PLANTACIONES EN PRODUCCIÓN}

Durante el periodo de Febrero a noviembre del 2004, en el Tercio inferior (TI) y Tercio superior (TS) de la planta no se registró posturas, ni huevos. Solo en el Tercio medio (TM) se encontró una postura con siete huevos en el mes de mayo a

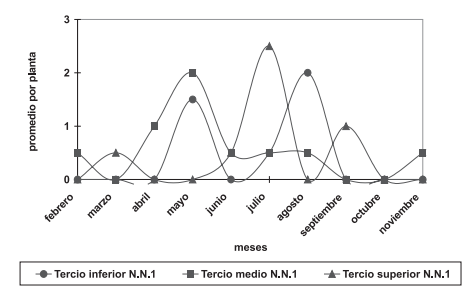

b

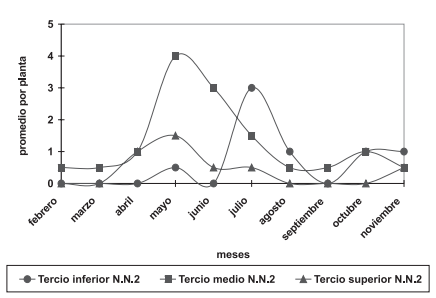

c
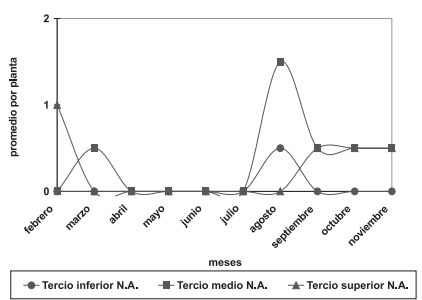

d

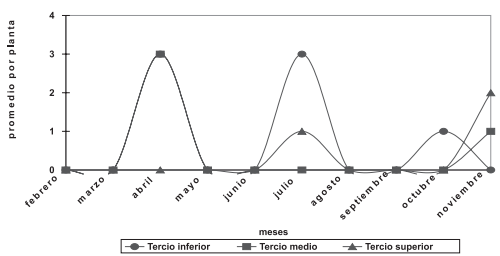

e

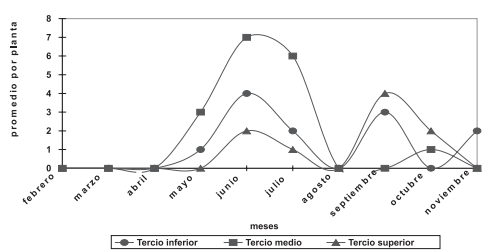

$f$

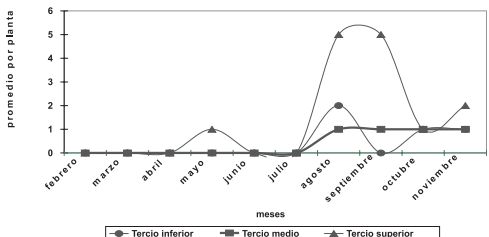

Figura 2 - $\mathrm{N}^{\circ}$ de de Edessa aff. aulacosterna en plantas de camu camu en el Distrito de Yarinacocha Pucallpa, Ucayali, Perú, durante el 2004. En producción: a. $\mathrm{N}^{\circ}$ de ninfas del I y II estadío (NN1). b. $\mathrm{N}^{\circ}$ de ninfas del III al $\checkmark$ estadío (NN2). c. $\mathrm{N}^{\circ}$ de adultos (NA). En desarrollo: d. $\mathrm{N}^{\circ}$ de ninfas del I y II estadío (NN1). e. $\mathrm{N}^{\circ}$ de ninfas del III al V estadío (NN2). f. $\mathrm{N}^{\circ}$ de adultos (NA). 
del 2004. la distribución espacial de posturas corresponde a un patrón de disposición con tendencia al azar (ID = 0,9; $\left.\mathrm{X}^{2}=0,03\right)$.

No se encontraron diferencias significativas en el número de ninfas I y II por mes entre el periodo seco $(2,1 \pm 1,7)$ y lluvioso $(1,0 \pm 0,0)(t=1,65 ; \mathrm{P}=0,15)$. Las ninfas del I y II estadío (NN1) presentaron en el TI (ID = 2,1; $\left.\mathrm{X}^{2}=27,8\right)$, TM $\left(\mathrm{ID}=1,5 ; \mathrm{X}^{2}=22,9\right)$ y TS $\left(\mathrm{ID}=1,6 ; \mathrm{X}^{2}=6,6\right)$ un patrón de disposición agregado. Asimismo, se observó un mayor número de ninfas en el TM (40 \%) (Figura 2a).

No se encontraron diferencias significativas en el número de ninfas III al $\mathrm{V}$ por mes entre el periodo seco $(3,3 \pm 1,9)$ y lluvioso $(1,5 \pm 0,5)(t=2,14 ; \mathrm{P}=0,07)$. La distribución espacial de las ninfas entre el III y V estadío (NN2) presentó en el TI $\left(\mathrm{ID}=1,7 ; \mathrm{X}^{2}=14,2\right), \mathrm{TM}\left(\mathrm{ID}=1,7 ; \mathrm{X}^{2}=27,2\right)$ y TS $(\mathrm{ID}=$ 1,$\left.1 ; \mathrm{X}^{2}=1,3\right)$ un patrón de disposición agregado. Asimismo, se observó una mayor presencia de ninfas en el TM $(54,1 \%)$ (Figura 2b).

No se encontraron diferencias significativas en el número de adultos por mes entre el periodo seco $(0,6 \pm 0,8)$ y lluvioso $(0,7 \pm 0,5)(t=0,20 ; P=0,84)$. La distribución espacial de los adultos en el TM (ID = 1,5; X $=9,4)$ y TS (ID = 1,1; X $=0,8$ ) muestra un patrón agregado, mientras que el TI (ID $\left.=0,9 ; \mathrm{X}^{2}=0,03\right)$ muestra un patrón al azar. Se observó una mayor presencia de adultos en el TM (54\%) (Figura 2c).

\section{PLANTACIONES EN DESARROLLO}

Durante el periodo de Febrero a noviembre del 2004 en el TI, TM y TS de la planta no se registró posturas de Edessa aff. Aulacosterna.

No se encontraron diferencias significativas en el número de ninfas I y II por mes entre el periodo seco $(0,8 \pm 1,6)$ y lluvioso $(2,2 \pm 2,8)(t=0,89 ; \mathrm{P}=0,41)$. La distribución espacial de las ninfas del I y II estadío (NN1) presentó en el TI (ID = 2,$\left.3 ; \mathrm{X}^{2}=40,4\right)$, TM $\left(\mathrm{ID}=2,3 ; \mathrm{X}^{2}=45,8\right)$ y TS $\left(\mathrm{ID}=1,5 ; \mathrm{X}^{2}=\right.$ $4,3)$ un patrón de disposición agregada; así mismo se observó un mayor número de ninfas en el TI (50\%) (Figura 2d).

Se encontraron diferencias significativas en el número de ninfas III al $\mathrm{V}$ por mes entre el periodo seco $(6,0 \pm 4,6)$ y lluvioso $(0,5 \pm 1,0)(\mathrm{t}=2,80 ; \mathrm{P}=0,03)$. La distribución espacial de las ninfas del III al V estadío (NN2) presentó en el TI (ID = 1,9; $\left.\mathrm{X}^{2}=5,5\right)$, TM (ID $\left.=2,7 ; \mathrm{X}^{2}=229,2\right)$ y TS (ID $\left.=1,6 ; X^{2}=6,6\right)$ un patrón de disposición agregado, así mismo se observó una mayor presencia de ninfas en el TM (45\%) (Figura 2e).

No se encontraron diferencias significativas en el número de adultos por mes entre el periodo seco $(3,0 \pm 3,3)$ y lluvioso $(1,0 \pm 2,0)(\mathrm{t}=1,18 ; \mathrm{P}=0,27)$. La distribución espacial de los adultos en el TI (ID $\left.=1,3 ; \mathrm{X}^{2}=1,9\right)$ y TS (ID = 3,3; $\mathrm{X}^{2}=$ 291,2) mostró un patrón agregado, mientras que en el TM $\left(\mathrm{ID}=0,8 ; \mathrm{X}^{2}=0,5\right)$ mostró un patrón al azar. Así mismo, se observó una mayor presencia de adultos en el TS (64\%) (Figura 2f).

El patrón de distribución agregado es el común para la mayoría de los insectos plaga (Malaguido \& Panizzi, 1999). Este patrón se ha encontrado mayormente en las ninfas y los adultos de Edessa aff. aulacosterna en plantaciones en producción y desarrollo. Mayormente no se encontró efecto del periodo seco y lluvioso sobre el número de individuos censados mensualmente de los estados ninfales y adultos en plantaciones en desarrollo y producción de camu camu. Pinedo et al. (2001) señalan que el sustrato donde se desarrolla el cultivo de camu camu como son los suelos inundables de restinga aseguran la mayor parte de los nutrientes requeridos. Por lo que los niveles de precipitación tendrían menor influencia como factor limitante para el cultivo. De esta forma Edessa aff. aulacosterna encontraría durante todo el año al cultivo de camu camu en condiciones adecuadas, no presentando diferencias marcadas en sus poblaciones entre los periodo secos y lluviosos. El que se encontrara un mayor número de ninfas del III al V estadio de Edessa aff. aulacosterna en la época seca en plantaciones de camu camu en desarrollo, pudiera explicarse porque el estadio $\mathrm{V}$ es considerado el más crítico dentro del desarrollo ninfal y durante este periodo el insecto necesita un alimento de alta calidad nutricional, y por ser plantación en desarrollo aún hay ausencia del fruto, y esto aunado a altos niveles de precipitación. Panizzi y Oliveira (1998) encontraron para el pentatomido Euchistus heros (Fabricius, 1794) que el tipo de alimento empleado como sustrato (Cajanus cajans (L.) Millsp. y Glycine $\max (\mathrm{L}$.) Merrill) influye en el tiempo de desarrollo y la mortalidad ninfal.

Sin embargo, se requiere mayores estudios que permitan explicar adecuadamente estos resultados.

No se encontraron diferencias en el número de ninfas I y II mensuales entre las plantaciones en producción $(1,7 \pm 1,4)$ y en desarrollo $(1,4 \pm 2,2)(t=0,37 ; \mathrm{P}=0,71)$. Tampoco, se encontraron diferencias en el número de ninfas III al $\mathrm{V}$ mensuales entre las plantaciones en producción $(2,6 \pm 1,7)$ y en desarrollo $(3,8 \pm 4,5)(\mathrm{t}=0,98 ; \mathrm{P}=0,35)$. En adición, no se vio diferencias en el número de adultos mensuales entre las plantaciones en producción $(0,7 \pm 0,6)$ y en desarrollo $(2,2 \pm 2,9)(t=1,93 ; P=0,08)$. El que no existieran diferencias en las poblaciones de Edessa en estadío ninfal y en adulto en plantaciones en producción y desarrollo, muestra que a pesar que este chinche se alimenta mayormente del fruto (característico de las plantaciones en producción), también puede alimentarse de las hojas tiernas (característico de las plantaciones en desarrollo) (Pinedo et al., 2001; Delgado \& Couturier, 2004). En contraste, para otros chinches pentatomidos como N. viridula, Acrosternum hilare (Say, 1831) y Euschistus servus (Say, 1832) en el cultivo de soya y algodón, y en $N$. viridula, Plautia affinis Dallas, 1851 
y Glaucias amyoti (Dallas, 1851) en Ligustrum lucidum Aiton (Oleaceae) se ha encontrado influencia del estado de desarrollo de la planta en sus dinámicas estacionales (Bundy \& McPherson, 2000; Coombs, 2004).

Por otro lado, la presencia de plantas invasoras en el cultivo de camu camu de por lo menos 35 especies, podrían ser lugares de refugio para este chinche, por lo que debería elucidarse el real rol de estas plantas en las fluctuaciones poblacionales de Edessa. Lamentablemente no se conoce cuales son las más importantes plantas invasoras que sirven de refugio para la chinche (Pinedo et al., 2001).

\section{COMPORTAMIENTO}

Este insecto se encuentra ampliamente distribuido en la amazonía peruana tanto en plantaciones como en rodales naturales, su actividad se inicia a partir de las 17:00 h, habiéndose observado una mayor actividad entre las 18:00 h y 19:00 h (Pinedo et al., 2001).

\section{AGRADECIMIENTOS}

Al Instituto de Investigaciones de la Amazonía Peruana (IIAP), que permitió que este trabajo fuera presentado como exposición oral en la XLVII Convención Nacional de Entomología realizada del 23 al 27 de Octubre del 2005 en la Ciudad de Ica, Perú. Al Dr. José A.M. Fernandes, Universidade Federal do Pará (UFPA), Brazil por la identificación de la especie de Edessa. La especie de Edessa del camu camu al parecer prontamente será descrita como una nueva especie por el Dr. Fernandes.

\section{BIBLIOGRAFÍA CITADA}

Barcello, A.; Grazia, J. 2003. Revision of Brachystethus (Heteroptera, Pentatomidae, Edessinae). Iheringia, Ser. Zool., 93: 413-446.

Bundy, C.S.; McPherson, R.M. 2000. Dynamics and seasonal abundance of stink bugs (Heteroptera: Pentatomidae) in a cotton-soybean ecosystem. J. Econ. Entomol., 93: 697-706.

Coombs, M. 2004. Broadleaf privet, Ligustrum lucidum Aiton (Oleaceae), a late-season host for Nezara viridula (L.), Plautia affinis Dallas and Glaucia amyoti (Dallas) (Hemiptera: Pentatomidae) in northern New South Wales, Australia. Aust. J. Entomol., 43: 335-339.

Correa-Ferreira, B.S.; de Azevedo, J. 2002. Soybean seed damage by different species of stink bugs. Agric. Forest Entomol., 4: $145-150$

Delgado, C.; Couturier, G. 2004. Manejo de insectos plagas en la Amazonía: su aplicación en camu camu. IIAP- Iquitos Perú/ IRDFrancia. Tarea Educación Gráfica Educativa Ed. 147 pp.
Jones, P.J.; Westcot, D. 2002. The effect of seasonal changes on Nezara viridula (L.) (Hemiptera: Pentatomidae) and Trissolcus basalis (Wollaston) (Hymenoptera: Scelionidae) in Hawaii. Biological Control, 23: 115-120.

Malaguido, A.B.; Panizzi, A.R. 1999. Nymph and adult biology of Euschistus heros (Hemiptera: Pentatomidae) and its abundance related to planting date and phenological stages of sunflower. Annals Entomol. Soc. America, 92: 424-429.

Nascimento, F.; De Oliveira, G.; Da Silva, N.M. 2003. Danos de Conotrachelus dubiae (Coleóptera: Curculionidae) em frutos de camu-camu (Myrciaria dubia) na Amazônia Central. Rev. Bras. Frutic., 25: 544-545.

Noda, T.; Kamano, S. 2002. Artificial rearing of Nezara viridula (L.) and $N$. antennata Scott (Heteroptera: Pentatomidae) with semisolid meridic diets. Appl. Entomol. Zool., 37: 43-50.

Panizzi, A.R.; Oliveira, E.D.M. 1998. Performance and seasonal abundance of the neotropical brown sitnk bug, shape Euschistus heros nymphs and adults on a novel food plant (pigeonpea) and soybean. Entomol. Exp. Appl., 88: 169-175.

Panizzi, A.R.; Vivan, L.M. 1997. Seasonal abundance of the neotropical brown stink bug, Euschistus heros, in overwintering sites, and the breaking of dormancy. Entomol. Exp. Appl., 82: 213-217.

Panizzi, A.R.; Cardoso, S.R.; Oliveira, E.D.M. 2000. Status of pigeonpea as an alternative host of Piezodorus guildinit (Hemiptera: Pentatomidae), a pest of soybean. Florida Entomol., 83: 334-342.

Panizzi, A.R. 2002. Stink bugs on soybean in Northeastern Brazil and a new record on the Southern green stink bug, Nezara viridula (L.) (Heteroptera: Pentatomidae). Neotrop. Entomol., 31: 331-332.

Perioto, N.W.; Lara, R.I.R.; Coutinho, D.; Milani, D. 2004. Pentatomídeos (Insecta, Hemiptera) fitófagos associados a gergelim Sesamum indicum L. (Pediliaceae) em Riberao Preto, SP, Brasil. Arq. Inst. Biol. Sao Paulo, 71: 93-94.

Pinedo, M.; Rita, R.; Rengifo, E.; Delgado, C.; Villacres, J.; Gonzáles, A.; Herminio, I.; López, A.; Ferronay, R.; Linares, C. 2001. Sistema de producción de camu camu en restinga. IIAP. Programa de Ecosistemas Terrestres. Proyecto Bioexport camu camu, Perú. $141 \mathrm{pp}$.

Riba, M.; Martí, J.; Sans, A. 2003. Influence of azadirachtin on development and reproduction of Nezara viridula L. (Het., Pentatomidae). J. Applied Entomol., 127: 37-41.

Rodrigues, R.B.; Menezes, H.C.; Cabral, L.M.C.; Dornier, M.; Reynes, M. 2001. An amazonian fruit with a high potential as a natural source of vitamin C: the camu-camu (Myrciaria dubia). Fruits, 56: 345-354. 
Sánchez, M.; Díaz, D.; Maselli, M. 1999. El comportamiento y tiempo de desarrollo de la chinche Edessa meditabunda (F) (Hemíptero; Pentatomidae). Rev. Fac. Agron. (Maracay), 25: 149-158.

Serra, G. V.; La Porta, N.C. 2001. Aspectos biológicos y reproductivos de Piezodorus guildinii (West.) (Hemiptera: Pentatomidae) en condiciones de laboratorio. Agriscientia, 18: 51-58.

Silva, E.J.E.; Fernandes, J.A.M.; Gracia, J. 2004. Variações morfológicas em Edessa rufomarginata e revalidação de $E$. albomarginata e E. marginalis (Heteroptera, Pentatomidae, Edessinae), Iheringia, Ser. Zool., 94: 261-268.

Sosa-Gomez, D.R.; Moscardi F. 1998. Laboratory and field studies on the infection of stink bugs, Nezara viridula, Piezodorus guildinii, and Euschistus heros (Hemiptera: Pentatomidae) with Metarhizium anisopliae and Beauveria bassiana in Brazil. J. Invertebr. Pathol., 71:115-20.
Todd, J.W. 1989. Ecology and behavior of Nezara viridula. Ann. Rev. Entomol., 34: 273-292.

Villachica, H. 1996. El cultivo de camu camu. (Myrciaria dubia H.B.K. Mc Vaugh), en la Amazonía peruana. TCA/SPT, Lima. Perú. 95pp.

Zar, J.H. 1996. Biostatistical analysis. Upper Saddle River, PrenticeHall Inc. Ed. 3. 662pp.

Recebido em 20/04/2006

Aceito em 18/09/2007 